\title{
Long term survival and late repair of massive cardiac pseudoaneurysm
}

\author{
YOAV TURGEMAN, * TIBERIO ROSENFELD, „ DAVID A HALON \\ From the Departments of Cardiology, *Central Emeq Hospital, Afula, and Lady Davis Carmel Hospital, \\ Haifa, Israel
}

SUMMARY Myocardial rupture of the left ventricle after an acute myocardial infarction resulted in the formation of a massive pseudoaneurysm in a middle aged man. Cross sectional echocardiography was useful in identifying the defect which was successfully closed at operation nine years after its formation.

Rupture of the left ventricular free wall during acute myocardial infarction usually leads to haemopericardium, cardiac tamponade, and sudden death. ${ }^{1}$ Long term survival without early surgical intervention is rare, ${ }^{2}$ but in some patients the rupture leads to the formation of a pseudoaneurysm. We report a patient who survived for an unusually long time after myocardial rupture and the formation of a massive pseudoaneurysm.

\section{Case report}

A 56 year old man presented with advanced congestive heart failure and recurrent pulmonary oedema. Nine years before he was admitted to hospital with acute transmural myocardial infarction. The electrocardiogram showed that the lateral wall was the site of infarction. The chest $x$ ray on admission showed a normal sized heart with mild pulmonary congestion. One week later the patient suffered repeated episodes of chest discomfort followed by sudden circulatory collapse, extreme cyanosis, and paroxysmal atrial fibrillation. Recurrent pulmonary embolism was diagnosed and he was treated by intravenous fluids and heparin. The chest $x$ ray showed progressive cardiac enlargement but there were no new electrocardiographic changes or an increase in cardiac enzyme activity. In the next nine years progressive heart failure developed which was characterised by recurrent episodes of pulmonary oedema. At admission physical examination showed

Requests for reprints to Dr David A Halon, Department of Cardiology, Lady Davis Carmel Hospital, 7 Michal Street, Haifa 34362, Israel. a blood pressure of $110 / 60 \mathrm{~mm} \mathrm{Hg}$ and atrial fibrillation with a ventricular response of $80-90$ beats/min. The jugular venous pressure was elevated to $20 \mathrm{~cm}$ above the sternal angle and showed a prominent $\mathrm{V}$ wave. There were signs of pulmonary hypertension and severe right heart failure with prominent ascites and anasarca. In addition, coarse rales were heard over both lungs. Examination of the heart revealed a dyskinetic apex that was displaced to the anterior axilliary line, an apical pansystolic murmur grade $3 / 6$ radiating to the axilla, and a third heart sound. A continuous to and fro murmur was present in the left midaxillary line in the third and fourth intercostal space. The electrocardiogram showed atrial fibrillation, left axis deviation, and signs of old lateral myocardial infarction (fig 1). The chest $x$ ray showed a considerably enlarged heart (cardiothoracic ratio of 0.65) and pulmonary venous hypertension. Fluoroscopy showed mild pericardial calcification along the left lateral cardiac border.

Echocardiography revealed a very large multiloculated pseudoaneurysm of the left ventricle communicating with the left ventricular cavity through a defect in the basal lateral myocardial wall (fig 2). The ventricle itself was moderately enlarged and there was reduced global contractility. There were no primary valve abnormalities.

At cardiac catheterisation the mean right atrial pressure was $12 \mathrm{~mm} \mathrm{Hg}(1.6 \mathrm{kPa})$, right ventricle $50 / 6-14 \mathrm{~mm} \mathrm{Hg}(6 \cdot 7 / 0 \cdot 8-1 \cdot 9 \mathrm{kPa})$, pulmonary artery $50 / 20 \mathrm{~mm} \mathrm{Hg}(6 \cdot 7 / 2 \cdot 7 \mathrm{kPa})$, and the wedge pressure was $26 \mathrm{~mm} \mathrm{Hg}(3.5 \mathrm{kPa})$ with a $\mathrm{V}$ wave of $40 \mathrm{~mm} \mathrm{Hg}$ $(5.3 \mathrm{kPa})$. The forward cardiac index was low $\left(1.6 \mathrm{l} / \mathrm{min} / \mathrm{m}^{2}\right)$. Selective coronary angiography demonstrated total occlusion of the left circumflex 


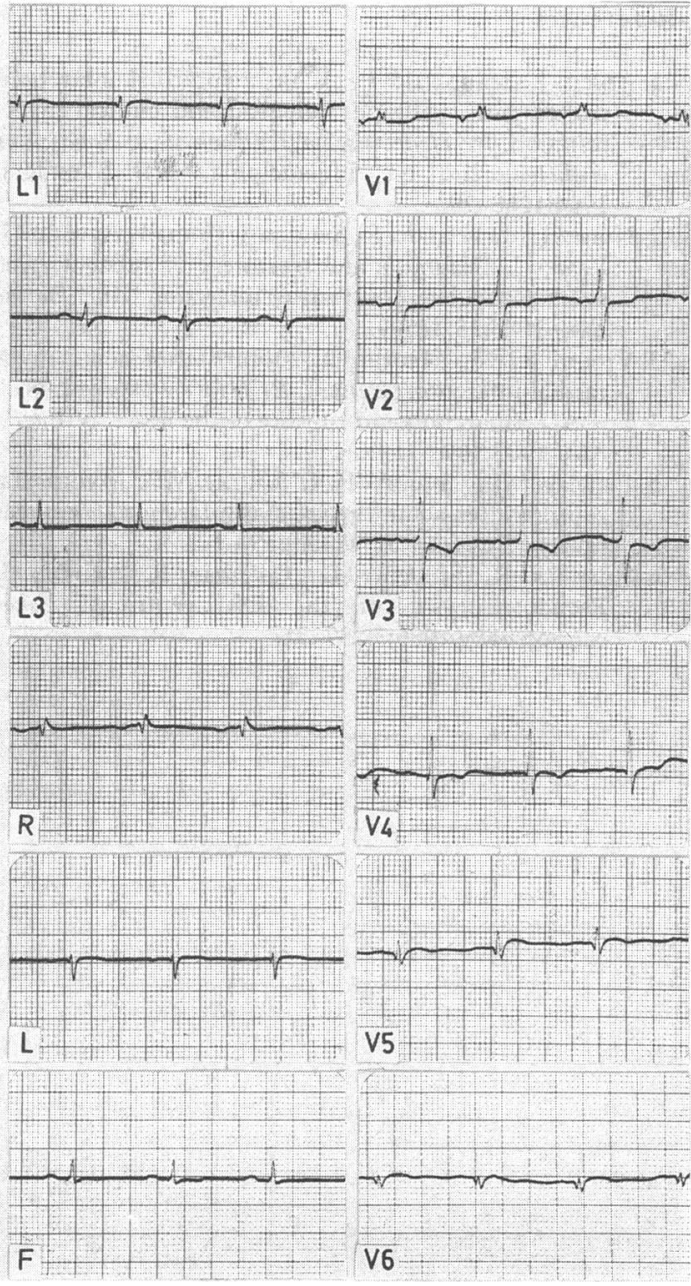

Fig 1 Electrocardiogram at admission. There are small $q$ waves and loss of $R$ waves in the lateral leads, indicating lateral wall infarction.

artery and a $60 \%$ narrowing of the proximal left anterior descending artery with no other important coronary disease. Left ventriculography showed large volumes of dye passing from the left ventricle into the pseudoaneurysm.

At operation a multiloculated pseudoaneurysm with numerous adhesions was found. The location of the myocardial rupture on the posterolateral aspect of the left ventricular free wall close to the posterior papillary muscle confirmed the echocardiographic appearance. The mitral valve itself was not directly affected by the pathological process. At operation the perforation was covered with a Teflon patch and after a rather long convalescence

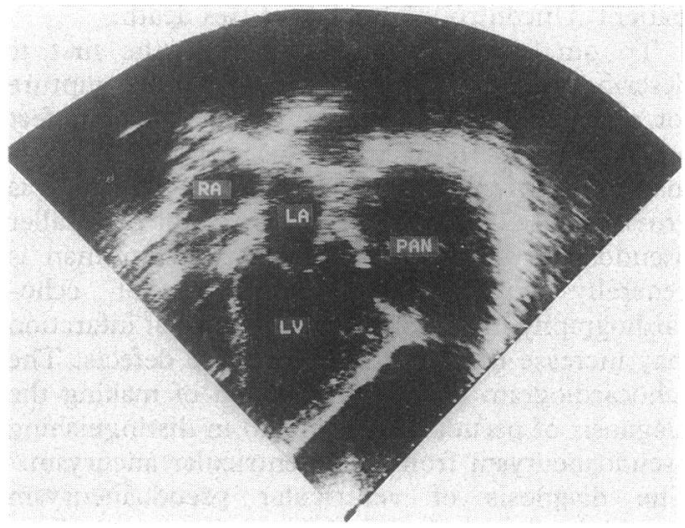

Fig 2 Cross sectional echocardiogram (apical four chamber view). There is a large multiloculated pseudoaneurysm $(P A N)$ which extends around the anterolateral wall of the left ventricle to its apex. The neck of the pseudoaneurysm communicates with the left ventricular cavity $(L V)$ through the discontinuity in the basal anterolateral wall. $R A$, right atrium; $L A$, left atrium.

the patient returned to daily activity and a sedentary occupation. Three months after operation he was limited only by particularly strenuous activity (New York Heart Association functional class II).

\section{Discussion}

Free wall rupture is believed to occur in $0.5-3.1 \%$ of patients after acute myocardial infarction. ${ }^{3}$ Myocardial rupture usually causes sudden death; the formation of a false aneurysm is quite rare. ${ }^{4}$ Without early surgical intervention long term survival of patients with myocardial rupture is rare $^{25}$ and most reports emphasise the need for very early recognition of this complication and immediate vigorous treatment. Pseudoaneurysm formation, while temporarily limiting the course of myocardial rupture, itself produces important haemodynamic complications, including a low cardiac output due to regurgitation of blood into the pseudoaneurysm (analogous to true cardiac aneurysms). In addition, true or functional incompetence may occur after malfunction of the mitral valve apparatus, and in patients with very large pseudoaneurysms the extra volume contained within the pericardium may add an element of compression which can simulate cardiac tamponade. Moreover, since the wall of the pseudoaneurysm is composed of clot, connective tissue, and fibrosis, this tissue may not contain the rupture permanently and it is common for recurrent leakage and sealing off of a pseudoaneurysm to produce the multiloculations-as were found in this 
patient. Uncontrolled leakage causes death.

To our knowledge this report is the first to describe a patient who survived myocardial rupture for nine years before surgical closure of the defect was performed. In this patient pseudoaneurysm formation was remarkedly advanced and there was gross cardiac disability. The formation of smaller pseudoaneurysms may be more common than is generally appreciated. Wider use of echocardiography in patients after myocardial infarction may increase the recognition of these defects. The echocardiogram is a useful method of making the diagnosis of pseudoaneurysm and in distinguishing pseudoaneurysm from true ventricular aneurysm. ${ }^{6}$ The diagnosis of ventricular pseudoaneurysm should be borne in mind in patients who have unusual physical signs, unusual cardiomegaly, or disproportionate cardiac failure after myocardial infarction.

\section{References}

1 Alpert J, Braunwald E. Acute myocardial infarction: pathological, pathophysiological and clinical manifestation. In: Heart disease, textbook of cardiovascular medicine. 2nd ed. Philadelphia: WB Saunders, 1984:1268-70.

2 Nunez L, de la Llana R, Sendon J, Coma I, Aguado G, Lawes J. Diagnosis and treatment of subacute free wall ventricular rupture after infarction. Ann Thorac Surg 1983;35:525-9.

3 Vroom RJAF. Myocardial rupture after acute myocardial infarction [Letter]. Br Heart $J$ 1984;51:98.

4 Fox AC. Infarction and rupture of the heart. $N$ Engl $J$ Med 1983;309:551-3.

5 Feneley MP, Chung VP, O'Rourke MF. Myocardial rupture after acute myocardial infarction. Ten year review. Br Heart J 1983;49:550-6.

6 Adamick R, Sprecher D, Coleman RE, Kisslo J. Pseudoaneurysm of the left ventricle. Echocardiography 1986;3:237-51. 